\title{
Parametrization of Spline Functions to Describe the Shape of Shell Structures ${ }^{* * *}$
}

\section{Introduction}

The traditional method of describing the shape of the surface of shell structures are the surfaces of the second degree (quadrics). Among the greatest disadvantages of this method is the lack of representation of local deformations of the structure and the dependence of the model shape of the position of each of the measured points.

It is much better to reproduce the actual shape of the surface using models based on spline functions, which eliminate the aforementioned disadvantages. These functions, however, are sensitive to other factors, the most important of which is related to the shape of the structure, determining an appropriate selection of the spline function arguments. Determining of the arguments is carried out in the so-called parametrization process, which is crucial for the accuracy of the obtained approximations. Besides parametrization, there are several additional factors which can be used to influence the shape of spline functions (boundary conditions, weighting of NURBS functions, smoothing using approximating functions). They are, however, of lesser significance for the parametrization, and their use often requires making subjective decisions. Boundary conditions concern only surface's periphery. For NURBS functions there are no tests allowing us to relate the weights with the magnitude of shape changing.

This paper focuses on the application and comparison of several popular parametrization methods for approximating the shape of the surface of shell structures. Comparisons were made for the two models: with one- and multi-directional variable curvature.

\footnotetext{
* AGH University of Science and Technology, Faculty of Mining Surveying and Environmental Engineering, Krakow, Poland

** Cracow University of Technology, Faculty of Environmental Engineering, Krakow

*** This work has been made within scientific research program no. 11.11.150.005
} 
The aim of the research is to identify the optimal parametrization method for describing shell structures, measured with a not too high density, for example, using popular reflectorless total stations.

\section{Basic Methods of Spline Function Parametrization}

Spline functions are usually presented in the B-spline representation $[2,4]$, which is a linear combination of base polynomials $N_{i}$ (defined with a recursive formula of Mansfield-de Boor-Cox (2)), with coefficients in the form of $d_{i}$ control points:

$$
S_{i}(t)=\sum_{i=0}^{n-m-1} d_{i} N_{i}^{m}(t) \quad t_{i}=t_{0}, t_{1}, \ldots, t_{n}
$$

where:

$t_{i}$ - nodes,

$d_{i}$ - control points,

$N_{i}$ - base polynomials,

$m$ - the degree of a function,

$n$ - the number of points:

$$
\begin{gathered}
N_{i}^{0}(t)= \begin{cases}1 & \text { for } t \in\left[t_{\mathrm{i}}, t_{i+1}\right) \\
0 & \text { for } t \notin\left[t_{\mathrm{i}}, t_{i+1}\right)\end{cases} \\
N_{i}^{k}(t)=\frac{t-t_{i}}{t_{i+k}-t_{i}} N_{i}^{k-1}(t)+\frac{t_{i+k+1}-t}{t_{i+k+1}-t_{i+1}} N_{i+1}^{k-1}(t)
\end{gathered}
$$

Selection of the nodes (arguments) $t_{i}$ of the function is carried out in the process of parametrization $[2,3,6]$ :

$$
\begin{aligned}
& t_{0}=0 \\
& t_{j}=t_{j-1}+\frac{T_{j}}{\sum_{i=1}^{n} T_{i}} \quad \text { for } t \in[0,1] \\
& t_{n}=t_{n-1}+\frac{T_{n}}{\sum_{i=1}^{n} T_{i}}=1
\end{aligned}
$$

where $T_{i}$ - the parameters. 
Currently, the most popular methods for determining the parameters $T_{i}$ belong to the family of exponential parametrization $[3,5]$ :

$$
T_{i}=\left(l_{i, i-1}\right)^{e} \quad \text { for } e \in[0,1]
$$

where $l_{i, j-1}$ - distances between points.

The simplest of them operates on a fixed unit distance $(e=0)$, bringing significant reduction in computation time. As it does not take into account the distance between the points, it may bring the distortion of the spline function for their uneven distribution:

$$
T_{i}=\text { const }
$$

Parametrization with the distance between points $(e=1)$ is recommended to describe objects of mild changes in their shape:

$$
T_{i}=l_{i, i-1}=\sqrt{\left(x_{i}-x_{i-1}\right)^{2}+\left(y_{i}-y_{i-1}\right)^{2}+\left(z_{i}-z_{i-1}\right)^{2}}
$$

Parametrization with the root of the distance between points $(e=0.5)$ is recommended to describe objects with significant changes in their shape:

$$
T_{i}=\sqrt{l_{i, i-1}}
$$

\section{Experiment Assumptions and Test Results}

The main task of test research was to determine the best parametrization method to describe the shape of the surfaces of shell structures, measured with an average density, for example, using popular reflectorless total stations. For this purpose, two ideal test models with curvature changing in one (elliptic cylinder) or more directions (three axial ellipsoid) were generated (Fig. 1). The horizontal span of the models is approximately $20 \mathrm{~m}$ and the vertical - about $15 \mathrm{~m}$. From both models, two alternately distributed sets of points were considered, the "measuring" set used for the construction of spline surfaces, and the "testing" set used to compare created surfaces with test models. Deviation on the "testing" points (in the direction normal to the model) measure the accuracy of determining the surface using a particular parametrization method. To create a spline surface, three parametrization methods described above were used, i.e. a fixed distance, the distance between the points and the root of the distance. The surfaces were created by the lofting method $[2,4]$ where spline curves are created based on the points, and then spreading spline surface patches on them:

$$
S_{i, j}(t, u)=\sum_{i=0}^{n-4} \sum_{j=0}^{r-4} d_{i, j} \cdot N_{i}^{3}(t) \cdot N_{j}^{3}(u) \quad t_{i}=t_{0}, t_{1} \ldots, t_{n} \quad u_{i}=u_{0}, u_{1} \ldots, u_{r}
$$


The nodes $t_{i}$ defining curves and $u_{i}$ defining surfaces were determined by the same parametrization method. Tests were conducted using Rhinoceros 4.0 software.

a)
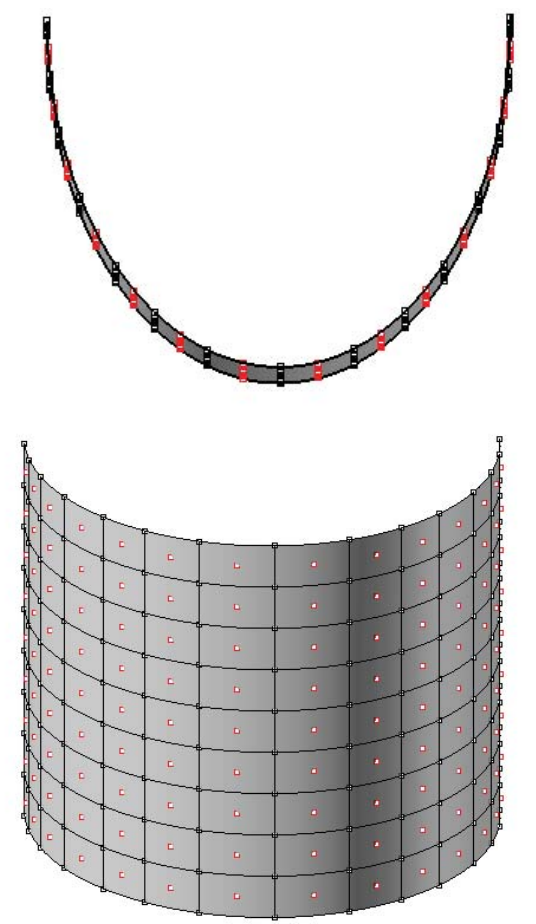

b)
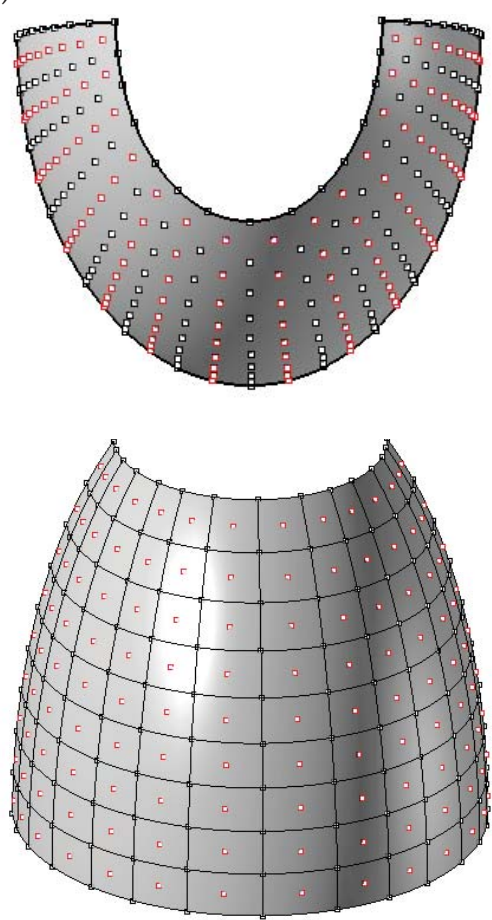

Fig. 1. Models used for testing with "measurement points" (grid nodes on lower figures) and "test" points (remaining points): a) single-curvature (elliptic cylinder);

b) multi-curvature (three axial allipsoid)

\subsection{Parametrization with a Fixed Distance}

Spline surfaces created using a fixed distance parametrization are characterized by the worst accuracy of reproducing the position of test points (Fig. 2). The mean deviation value for the one-curvature model is $6.6 \mathrm{~mm}$, with the maximum values reaching $16 \mathrm{~mm}$. The corresponding deviation values for the multi-curvature model are $7.3 \mathrm{~mm}$ and $30 \mathrm{~mm}$, respectively.

Both models are characterized by a similar value of the mean deviations, with twice the difference of maximum deviations. It results from the disproportion of the point density, dependent upon the curvature of a model. The multi-curvature model, having larger local curvatures than the one-curvature model, also has larger local deviation values at the "testing" points. However, in the upper part of the 
model, the points forming a spline surface, due to the curvature of the model, are more densely distributed, which results in a better approximation, limiting mean deviation values of the whole model. According to the papers [1, 4], doubling the density of the points forming a spline function will improve the accuracy of approximation four times:

$$
\varepsilon=|f-S| \leq \frac{1}{2} \max \left(l_{i, i-1}^{2}\right) M \quad \text { for } \quad i=0, \ldots, n
$$

where:

$$
\varepsilon \text { - error of approximation, }
$$

$f$ - approximating function,

$S$ - spline function,

$l_{i, j-1}$ - distance between points,

$$
M=\text { const and } M \geq\left|f^{\prime \prime}(x)\right| \text {. }
$$

A similar situation occurs in the case of the remaining types of parametrization.
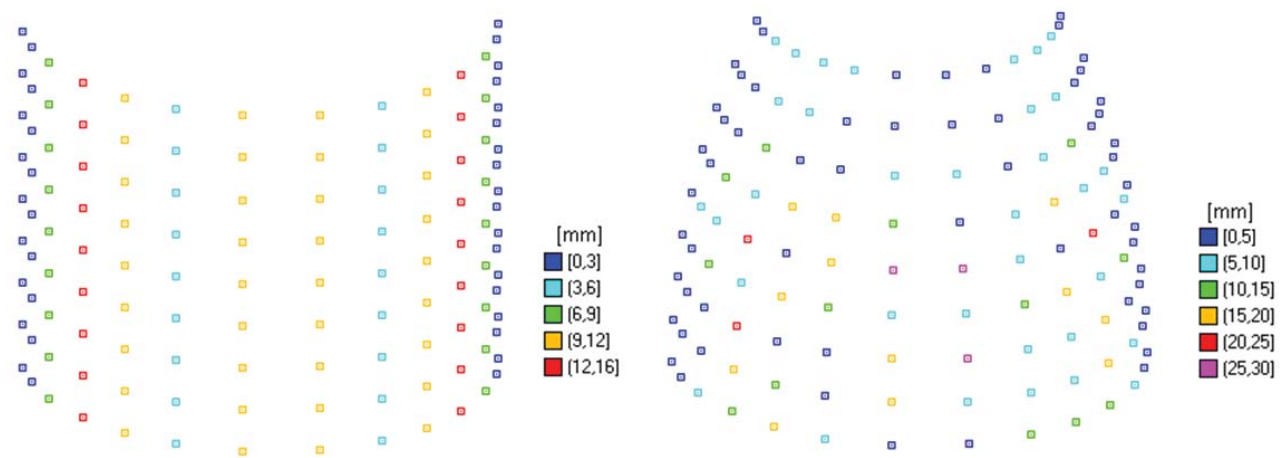

Fig. 2. Deviations of a spline surface parametrized with a fixed distance

Due to the fact that the fixed distance parametrization does not take into account a distance between the points, and the "measuring" points adopted for the construction of a spline surface are not equidistant (Fig. 1 - upper figures), the deviations occurred in an unpredictable manner, which is characteristic of this type of parametrization. This phenomenon is particularly noticeable for the multi-curvature model because the one-curvature model lacks variation in the vertical direction. The deviation values, from the point of view of deformation analysis of shell structures, are at a significant level (a few centimeters), particularly taking into account maximum deviations of a multi-curvature structure. It should be noted, that these are deviations only resulting from the applied method of approximation, while in practical applications additionaly appears the measurement error (usually several $\mathrm{mm}$ ). The overall accuracy of the surface modelled in this way may be in many cases unsatisfactory. 


\subsection{Parametrization with a Root of the Distance between Points}

Spline surfaces created using parametrization with a root of the distance between points has a mean accuracy of reproducing the position of "testing" points (Fig. 3). The mean deviation value for the one-curvature model is $3.5 \mathrm{~mm}$, with the maximum values reaching $8 \mathrm{~mm}$. The corresponding deviation values for the multicurvature model are $4.7 \mathrm{~mm}$ and $25 \mathrm{~mm}$, respectively.
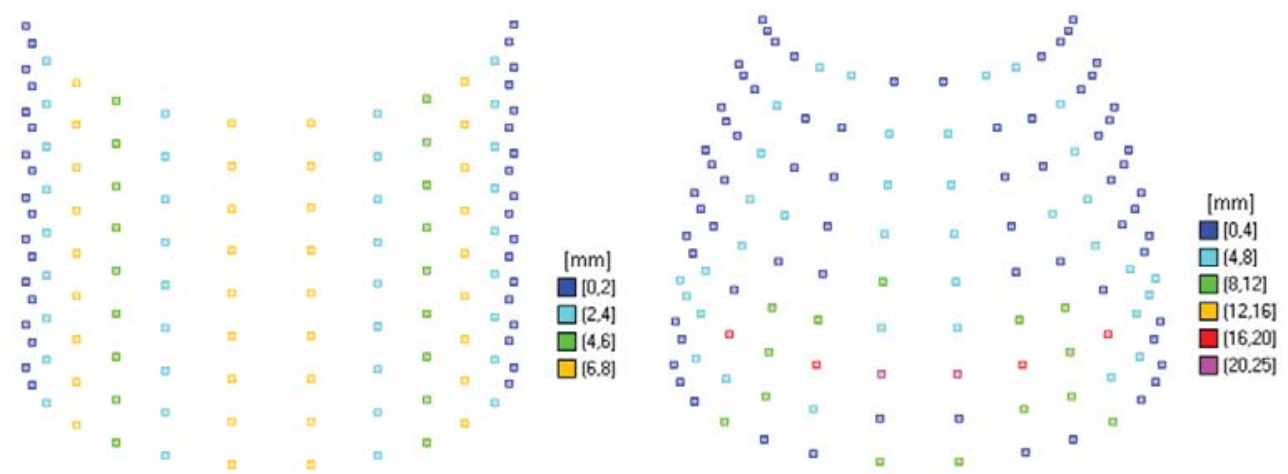

Fig. 3. Spline surface deviations parametrized with a root of the distance between points

For the one-curvature model there was a general, about double improvement in the accuracy of the results comparing to the parametrization with a fixed distance. For the multi-curvature model, a few points have significant deviations reaching $25 \mathrm{~mm}$, however, the mean deviation was significantly reduced compared to the parametrization with a fixed distance. As it was mentioned in section 2, the parametrization with a root of the distance between the points is recommended to describe objects with rapid changes of the shape. It protects against adverse fluctuations of the function, while causing flattening of the curve bends. The considered models, like most construction shell structures, are characterized by a relatively mild change in curvature. Therefore, there is no risk of loops or waving of the function. However, flattening of the curve bends will result in deterioration of the results accuracy, which will be the larger, the greater the distance between the points used to construct the surface. For the one-curvature model, the greatest distance between the "measuring" points were found between their vertical rows (the fourth and the fifth from the edge, and the middle ones (Fig. 1)). In the same areas, the largest deviations on the "testing" points occurred. For the multi-curvature model this phenomenon is clearly noticeable only in one, horizontal row of the "testing" points, due to the vertical curvature compensating for differences in distances for other areas of the surface. General deviation values were markedly reduced in comparison with the fixed distance parametrization. However, due to a failure to match parametrization with a root of distance to the shape of the analyzed structures, the results still can 
not be considered desirable, especially when considering the maximum deviations of the multi-curvature model.

\subsection{Parametrization of the Distance between Points}

Spline surfaces created using parametrization with the distance between points are characterized by the best accuracy of reproducing the position of the "testing" points (Fig. 4). The mean value of deviations for the one-curvature model is $0.8 \mathrm{~mm}$, with maximum values reaching $4 \mathrm{~mm}$. The corresponding deviation values for the multi-curvature model are $0.7 \mathrm{~mm}$ and $5 \mathrm{~mm}$, respectively. For both models, the largest deviations occurred in the places of significant curvature of the surface. For the multi-curvature model, the upper parts of the model, despite a greater curvature than the bottom ones, show smaller deviations because the vertical curvature reduced the distance between the "measuring points", which improved the accuracy of the surface. As it was mentioned in section 2, parametrization with the distance between points is recommended to describe objects with mild changes of the shape. It allows to highlight the function curves, which positively affected the accuracy of reproducing the "testing" points.
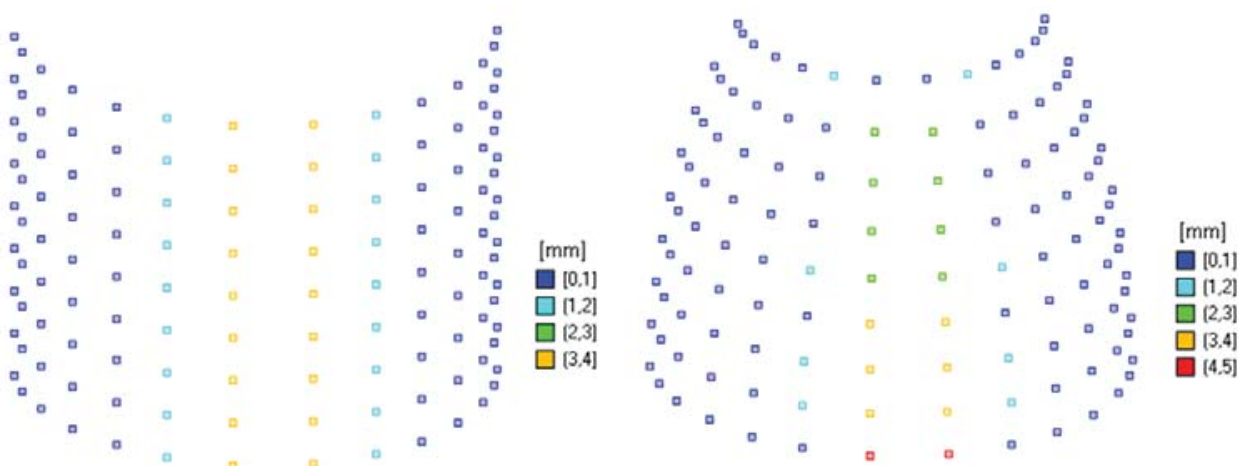

Fig. 4. Spline surface deviations parametrized with the distance between points

Maximum deviations are equal to or less than the mean values of measurement errors, obtained by the reflectorless method (a few milimeters). Mean deviations can be considered to be negligible in the analysis of the shape of shell structures.

\section{Conclusions}

The conducted studies revealed a significant influence of the applied parametrization method to the accuracy of determining a spline surface. The combined results from all methods in the uniform range of deviations were gathered in Table 1 . It 
contains the percentage of deviations falling within given ranges. The final results should be evaluated in terms of approximating the shape of the construction shell structures measured with a not too high density (e.g., using reflectorless total stations). Parametrization with a fixed distance gives the worst results of approximations, the deviations reach the highest values and their distribution is unpredictable. Parametrization with a root of the distance between points allows us to significantly reduce the deviation values which appear in predictable locations.

Table 1. Summary of deviations for all types of parametrization: $\mathrm{m} 1$ - single curvature model, $\mathrm{m} 2$ - multi curvature model

\begin{tabular}{|c|c|c|c|c|c|c|}
\hline \multirow{2}{*}{$\begin{array}{l}\text { Deviation } \\
{[\mathrm{mm}]}\end{array}$} & \multicolumn{2}{|c|}{$T_{i}=l_{i, i-1}$} & \multicolumn{2}{|c|}{$T_{i}=\sqrt{l_{i, i-1}}$} & \multicolumn{2}{|c|}{$T_{i}=$ const } \\
\hline & m1 [\%] & $\mathrm{m} 2[\%]$ & $\mathrm{m} 1[\%]$ & $\mathrm{m} 2[\%]$ & $\mathrm{m} 1[\%]$ & $\mathrm{m} 2[\%]$ \\
\hline [0.3] & 85.7 & 93.7 & 57.1 & 49.2 & 28.6 & 31.7 \\
\hline (3.6] & 14.3 & 6.3 & 14.3 & 25.4 & 14.3 & 20.6 \\
\hline (6.9] & - & - & 28.6 & 12.7 & 14.3 & 17.5 \\
\hline$(9.12]$ & - & - & - & 7.9 & 28.6 & 11.1 \\
\hline (12.15] & - & - & - & 0.0 & 14.3 & 3.2 \\
\hline (15.18] & - & - & - & 1.6 & - & 9.5 \\
\hline (18.21] & - & - & - & 1.6 & - & 1.6 \\
\hline (21.24] & - & - & - & 0.0 & - & 1.6 \\
\hline (24.27] & - & - & - & 1.6 & - & 1.6 \\
\hline (27.30] & - & - & - & - & - & 1.6 \\
\hline $\begin{array}{c}\text { Average dev. } \\
{[\mathrm{mm}]}\end{array}$ & 0.8 & 0.7 & 3.5 & 4.7 & 6.6 & 7.3 \\
\hline
\end{tabular}

By far the best accuracy results are achieved by parametrization with the distance between points. The deviation values are similar to those of average errors of the reflectorless measurement methods or smaller. This method should therefore be recommended to describe the shape of the surfaces of shell structures.

\section{References}

[1] Ahlberg J.H., Nilson E.N., Walsh J.L.: The theory of splines and their applications. Academic Press, New York 1967.

[2] Farin G.: Curves and surfaces for computer aided geometric design. Academic Morgan Kaufmann Publishers, San Francisco 2002.

[3] Haron H., Rehman A., Adi D.I.S., Lim S.P., Saba T. : Parameterization Method on B-Spline Curve. Mathematical Problems in Engineering, 2012. 
[4] Kiciak P.: Podstawy modelowania krzywych i powierzchni. WNT, Warszawa 2000.

[5] Lee E.T.Y.: Choosing nodes in parametric curve interpolation. Computer-Aided Design, vol. 21, no. 6, 1989, pp. 363-370.

[6] Lim C.G.: A universal parametrization in B-spline curve and surface interpolation. Computer Aided Geometric Design, vol. 16, no. 5, 1999, pp. 408-422. 